\title{
The RCAN1.4-calcineurin/NFAT signaling pathway is essential for hypoxic adaption of intervertebral discs
}

Bao Huang ${ }^{1,2}$, Yongqing He ${ }^{3}$, Shengwen $\mathrm{Li}^{3}$, Xiaoan Wei ${ }^{1,2}$, Junhui Liu ${ }^{1,2}$, Zhi Shan ${ }^{1,2}$, Yue Huang ${ }^{1,2}$, Jian Chen ${ }^{1,2}$ and Fengdong Zhao ${ }^{1,2}$

\begin{abstract}
Calcipressin-1, also known as regulator of calcineurin 1 (RCAN1), can specifically bind calcineurin at or near the calcineurin A catalytic domain and downregulate calcineurin activity. However, whether RCAN1 affects the hypoxic intervertebral disc (IVD) phenotype through the calcineurin/NFAT signaling pathway remains unclear. First, we confirmed the characteristics of the degenerative nucleus pulposus (NP) by H\&E, safranin O/fast green and Alcian blue staining, and detected increased RCAN1 levels in the degenerative NP by immunohistochemistry. Then, we demonstrated that the protein level of RCAN1.4 was higher than that of RCAN1.1 and progressively elevated from the control group to the Pfirmann grade $V$ group. In vitro, both hypoxia $\left(1 \% \mathrm{O}_{2}\right)$ and overexpression of HIF-1a reduced the protein level of RCAN1.4 in rat NP cells in a dose- and time-dependent manner. We further found that miRNA-124, through a nondegradative pathway (without the proteasome or lysosome), suppressed the expression of RCAN1.4. As expected, calcineurin in NP cells was activated and primarily promoted nuclear translocation of NFATC1 under hypoxia or RCAN1.4 siRNA transfection. Furthermore, SOX9, type II collagen and MMP13 were elevated under hypoxia, RCAN1.4 siRNA transfection or NFATc1 overexpression. Using chromatin immunoprecipitation (ChIP) and a luciferase reporter assay (with mutation), we clarified that NFATc1 increasingly bound the SOX9 promotor region (bp -367 -357). Interaction of HIF-1a and NFATc1 promoted MMP13 transcription. Finally, we found that FK506 reversed hypoxia-induced activation of the calcineurin/NFAT signaling pathway in NP cells and an ex vivo model. Together, these findings show that the RCAN1.4-calcineurin/ NFAT signaling pathway has a vital role in the hypoxic phenotype of NP cells. RCAN1.4 might be a therapeutic target for degenerative disc diseases.
\end{abstract}

\section{Introduction}

Intervertebral disc degeneration (IVDD) is a leading cause of low back pain (LBP), one of the most common clinical complaints ${ }^{1,2}$. Intervertebral discs (IVDs) include the central hydrated chondrocyte-like nucleus pulposus

\footnotetext{
Correspondence: Jian Chen (chenjian-bio@zju.edu.cn) or

Fengdong Zhao (zhaofengdong@zju.edu.cn)

${ }^{1}$ Department of Orthopedic Surgery, Sir Run Run Shaw Hospital, Zhejiang

University School of Medicine, Hangzhou, China

${ }^{2}$ Key Laboratory of Musculoskeletal System Degeneration and Regeneration

Translational Research of Zhejiang Province, Hangzhou, China

Full list of author information is available at the end of the article

These authors contributed equally: Bao Huang, Yongqing $\mathrm{He}$
}

(NP), which is inferiorly and superiorly bounded by the cartilaginous endplate (CEP) and laterally surrounded by the concentric lamellae of the annulus fibrosus (AF). IVDs are a complex tissue that accommodates high biomechanical forces and permits a range of motions between adjacent vertebrae ${ }^{3}$. Notably, the high proteoglycan content in the central water-binding NP is an important component of IVDs, which absorb stress and maintain the structure and function of the spine ${ }^{4}$. In addition, because of injury and inflammatory factors infiltration, imbalance between extracellular matrix anabolism and catabolism in the NP can lead to degenerative

\section{(c) The Author(s) 2020}

(c) (i) Open Access This article is licensed under a Creative Commons Attribution 4.0 International License, which permits use, sharing, adaptation, distribution and reproduction cc) in any medium or format, as long as you give appropriate credit to the original author(s) and the source, provide a link to the Creative Commons license, and indicate if changes were made. The images or other third party material in this article are included in the article's Creative Commons license, unless indicated otherwise in a credit line to the material. If material is not included in the article's Creative Commons license and your intended use is not permitted by statutory regulation or exceeds the permitted use, you will need to obtain permission directly from the copyright holder. To view a copy of this license, visit http://creativecommons.org/licenses/by/4.0/. 
disc diseases ${ }^{5}$. Human IVDs are the largest nonvascular tissue in the human body ${ }^{6,7}$. Therefore, the central NP is completely hypoxic and expresses hypoxia inducible factor 1 alpha and 2 alpha (HIF- $1 \alpha$ and HIF- $2 \alpha)^{8}$. HIF- $1 \alpha$ promotes ECM synthesis in NP and maintains the intracellular $\mathrm{pH}$ and viability of NP cells ${ }^{9}$. However, the protein level of HIF- $1 \alpha$ in the degenerative disc is generally reduced $^{10-12}$.

Calcipressin-1, also known as regulator of calcineurin 1 $(\mathrm{RCAN} 1)^{13}$, is in a family of negative endogenous regulators of calcineurin activation and can specifically bind the calcineurin A catalytic domain and downregulate calcineurin activity ${ }^{14,15}$. Calcineurin, composed of calcineurin A (the catalytic domain, $\mathrm{CnA}$ ) and calcineurin $\mathrm{B}$ (the binding domain, $\mathrm{CnB}$ ), is a protein phosphatase enzyme that primarily promotes the nuclear translocation of NFAT. The RCAN1 gene includes seven exons, and alternative splicing generates two main isoforms: RCAN1.1 (252 amino acids, 39 kDa) and RCAN1.4 (192 amino acids, $29 \mathrm{kDa}$ ). Recent studies showed that RCAN1.4 has a critical role in cancer growth ${ }^{16,17}$, endothelial cells migration ${ }^{18,19}$ and neuronal apoptosis ${ }^{20}$. Another study showed that prolyl hydroxylase domain 2 (PHD2) deficiency, which contributed to the limited oxygen-dependent degradation of HIF- $1 \alpha$ and might serve as a chronic HIF- $1 \alpha$ stabilization model, promotes skeletal muscle fiber repair through a calcineurin/NFATc1dependent pathway ${ }^{21,22}$. However, whether RCAN1.4 affects the hypoxic IVD phenotype through the calcineurin/NFAT signaling pathway remains unclear.

Previous studies showed that the calcineurin/ NFATc1 signaling pathway regulates sex-determining region Y (SRY)-box 9 (SOX9) expression during tracheal chondrogenesis $^{23}$ and that NFATc1 can induce SOX9 transcription in the pancreas and chondrocytes ${ }^{23-25}$. SOX9 is vital for aggrecan and typeIIcollagen gene expression. Furthermore, HIF- $1 \alpha$ is a positive regulator of SOX9 activity $^{22,26}$ and metabolically controls collagen synthesis and modification in chondrocytes ${ }^{27}$. In addition, HIF- $1 \alpha$ is upregulated by a process that involves calcineurin/NFAT in activated mast cells ${ }^{28}$, and HIF- $1 \alpha$ participates in the activation of calcineurin/NFAT ${ }^{29}$. However, the roles of RCAN1 in ECM synthesis and remodeling of the IVD have not been elucidated. Therefore, we hypothesize that the interaction between HIF-1 $\alpha$ and the RCAN1.4-calcineurin/ NFAT signaling pathway promotes ECM synthesis and remodeling to preserve the IVD phenotype.

To test our hypothesis, we detected the gene and protein expression levels of RCAN1.1 and RCAN1.4 in human NP specimens and analyzed their correlation with disc degeneration. Further, we evaluated the role of the RCAN1.4-calcineurin/NFAT signaling pathway in the hypoxic NP cell phenotype and silenced RCAN1.4 to detect its role in the NP cells. Moreover, we determined that FK506 (a calcineurin inhibitor) repressed hypoxiainduced activation of the calcineurin/NFAT signaling pathway using in vitro and ex vivo models.

\section{Materials and methods}

\section{Human tissue from normal and degenerated NP}

Informed consent was obtained from each patient. Our study was approved by the Ethical Review Board of Sir Run Run Shaw Hospital. From June 2018 to July 2019, human lumbar NP tissues were obtained from patients with vertebral fractures who did not present degenerative changes based on MR images, or patients with LBP who were undergoing discectomy and fusion. The patient information is listed in Supplemental Table 1. According to the Pfirrmann grading system ${ }^{30}$, the lumbar disc specimens were further subdivided into Pfirrmann grade I/II, grade III, grade IV, and grade V groups. Specimens were carefully dissected and either fixed in $4 \%$ paraformaldehyde for $48 \mathrm{~h}$ at $4{ }^{\circ} \mathrm{C}$ or immediately frozen in liquid nitrogen.

\section{NP cell isolation and treatment}

The animal experiments were approved by the Ethical Review Board of Sir Run Run Shaw Hospital (affiliated with Zhejiang University, Hangzhou, Zhejiang). All Sprague-Dawley rats (male, 150-200 g, 8-week-old) used were provided by the Animal Center of Zhejiang University (Zhejiang, China). Human or rat NP tissues were isolated from lumbar discs and then cut into small pieces $\left(<1 \mathrm{~mm}^{3}\right.$ in size $)$ as quickly as possible as previously described $^{31}$. After incubation with type II collagenase $(0.2 \mathrm{mg} / \mathrm{mL})$ (Sigma-Aldrich, USA) for $2 \mathrm{~h}$ at $37^{\circ} \mathrm{C}$, the cells were harvested and centrifuged at $800 \mathrm{rpm}$ for 5 minutes. The cells were resuspended with Dulbecco's modified Eagle's medium with $20 \%$ fetal bovine serum (Gibco, USA), $1 \mathrm{~mm}$ sodium pyruvate, $2 \mathrm{~mm}$ glutamine, and $1 \%$ penicillin/streptomycin and cultured in a humidified atmosphere containing $5 \% \mathrm{CO}_{2}$ at $37^{\circ} \mathrm{C}$. RCAN1 or HIF-1 $\alpha$ expression was evaluated by treating NP cells with hypoxia, dimethyloxalylglycine (DMOG, $0.5 \mu \mathrm{m}$ and $1 \mu \mathrm{m}$ ) or RCAN1.4 siRNA (Vigenebio, Shandong, China). The hypoxic incubator contained $1 \% \mathrm{O}_{2}, 5 \% \mathrm{CO}_{2}$, and $94 \%$ $\mathrm{N}_{2}$. In brief, using Lipofectamine $3000, \mathrm{NP}$ cells at an up to approximately $70 \%$ confluence were transfected with negative control siRNA or target siRNA (100 nM) (Invitrogen, CA, USA). To mimic hypoxia, we stimulated NP cells with DMOG (a competitive PHD inhibitor) (SigmaAldrich) for the indicated duration. Following the treatment, we used TRIzol reagent (Invitrogen) or RIPA (Gibco, Gaithersburg, MD, USA) to extract total RNA or proteins.

\section{RNA isolation and quantitative RT-PCR}

Human NP tissues were finely powdered with a sterilized mortar and pestle. Total RNA was extracted using 
the Ultrapure RNA Kit (CW0581, CWBIO, China). A NanoDrop 2000 was used to measure RNA quantity. Complementary DNA was synthesized using PrimeScript RT MasterMix (Takara Bio, Otsu, Japan). SYBR Green qPCR Master Mix (Takara Bio, Otsu, Japan) was used to quantify transcript levels. Sequences of the RT-qPCR primers are summarized in Supplemental Table 2. Experimental reactions were conducted by preincubation $\left(95^{\circ} \mathrm{C}\right.$ for $\left.5 \mathrm{~min}\right)$, amplification $\left(95^{\circ} \mathrm{C}\right.$ for $15 \mathrm{~s}, 60^{\circ} \mathrm{C}$ for $60 \mathrm{~s}$ for 40 cycles), melting curve analysis $\left(95^{\circ} \mathrm{C}\right.$ for $15 \mathrm{~s}$, $60^{\circ} \mathrm{C}$ for $60 \mathrm{~s}$ ), and cooling ( $40^{\circ} \mathrm{C}$ for 5 minutes). RT-qPCR was performed in triplicate, and amplification signals were normalized to the $\beta$-actin signal in the same reaction.

\section{Western blotting}

Following treatment, ground NP tissues or NP cells were immediately placed on ice and incubated for $\sim 40 \mathrm{~min}$ in RIPA buffer (Gibco) with $100 \mathrm{~mm}$ phenylmethanesulfonyl fluoride (PMSF) (Beyotime, Zhengzhou, China) and protease inhibitor cocktail (PIC) (Millipore, USA), followed by centrifugation at $12,000 \times g$ for $15 \mathrm{~min}$. Proteins were resolved by $10 \%$ sodium dodecyl sulfate-polyacrylamide gel electrophoresis (SDS-PAGE) and transferred to polyvinylidene difluoride membranes (Millipore, Billerica, MA) by electroblotting. The membranes were incubated in $5 \%(\mathrm{w} / \mathrm{v})$ nonfat dry milk in TBST (1\% Tween20) at RT for $1-2 \mathrm{~h}$ and then incubated with anti-RCAN1 (ab140131, Abcam, Cambridge, MA; abs121164, absin, China), anti-HIF-1 $\alpha$ (36169, CST; R1510-5, Huabio, Hangzhou), anti-SOX9 (ab185966, Abcam; ET1611-56, Huabio), anti-MMP13 (ab39012, Abcam), anti-type II collagen (ab34712, Abcam), antiNFATc1 (8032, CST; ET1704-45, Huabio), anti-histone H3 (4499, CST), anti-ubiquitin (3936, CST), anti-tubulin- $\alpha$ (Proteintech, USA), or anti- $\beta$-actin (Beyotime) and immunoglobulin $\mathrm{G}$ antibodies (1:1000 dilution) at $4{ }^{\circ} \mathrm{C}$ overnight. After $5 \times 7 \mathrm{~min}$ of TBST washing, protein bands were incubated with horseradish peroxidase (HRP)-conjugated goat anti-rabbit/mouse immunoglobulin G (1:5000 dilution; CST), followed by detection using electrochemical luminescence reagent (Millipore, Billerica, MA). Bands were detected with Image Lab software (Bio-Rad, Hercules, CA). The obtained images were measured with the ImageJ software (National Institutes of Health, Bethesda, MD, USA)

\section{Immunohistochemistry (IHC)}

Specimens were embedded in paraffin and sliced into $4 \mu \mathrm{m}$-thick sections. IHC was conducted with an SP Rabbit \& Mouse HRP Kit (CW2069, CWBIO). To investigate the immunoreactivity of RCAN1 (ab140131, Abcam) or HIF$1 \alpha$, slices were immunostained at $4{ }^{\circ} \mathrm{C}$ overnight. Slices were incubated with rabbit anti-RCAN1 or anti-HIF-1 $\alpha$ polyclonal antibodies and immunoglobulin G diluted 1:100 or 1:200. Three pathologists blinded to the clinical tissue data were responsible for counting the total cells and RCAN1- or
HIF-1 $\alpha$-positive cells in three sections of each specimen at a high-power field (magnification $\times 200$ ). The sections were recounted if the intraclass correlation coefficient was below 0.8 .

\section{Immunofluorescence staining}

Cells were placed on slides in six-well plates at a density of $1 \times 10^{4}$ cells. After incubation overnight, the cells were stimulated with or without hypoxia or FK506 for the indicated duration. The slides were then fixed in $4 \%$ paraformaldehyde for 30 minutes and permeabilizated with $0.5 \%$ Triton-X100 for 30 minutes at RT. After incubation with $5 \%$ bovine serum albumin for $1 \mathrm{~h}$, the slides were incubated with anti-SOX9, anti-type II collagen and anti-NFATc1 (1:100 200 dilution) at $4{ }^{\circ} \mathrm{C}$ overnight. Nuclei were stained with $0.1 \mu \mathrm{g} / \mathrm{mL}$ DAPI (Sigma-Aldrich) for 30 minutes at RT. Cells were imaged using fluorescence microscope model BX51TRF (Olympus Corporation, Tokyo, Japan). Images were analyzed by ImageJ software (National Institutes of Health).

\section{Immunoprecipitation (IP) assay}

In brief, cellular lysates were immunoprecipitated into $2 \mu \mathrm{g}$ of anti-RCAN1 or anti-Flag antibody (0912-1, Huabio) at $4{ }^{\circ} \mathrm{C}$ overnight, followed by incubation with $30 \mu \mathrm{L}$ of protein A/G-agarose $(50 \% \mathrm{v} / \mathrm{v})$ for $3 \mathrm{~h}$ at $4{ }^{\circ} \mathrm{C}$. After centrifugation at $13,000 \times g$ for $12 \mathrm{~min}$, protein complexes were harvested. After five washes with cold PBS, bound proteins were resolved by $10 \%$ SDS-PAGE and finally incubated with anti-Myc (R1208-1, Huabio) and antiubiquitin (3936, CST).

\section{Chromatin Immunoprecipitation (ChIP)}

In brief, using a ChIP kit (9002, CST), NP cells were stimulated with or without hypoxia for $8 \mathrm{~h}$, followed by incubation in $37 \%$ formaldehyde for 10 minutes, as described previously ${ }^{32}$. After cross-linking was terminated with glycine and the cells were washed $3 \times 10$ minutes using cold PBS with $1 \mathrm{~mm}$ PMSF and PIC, cells were harvested, resuspended in ChIP buffer and incubated for 30 minutes. The cells were then sonicated via 10 cycles consisting of 30-s pulses and 30-s intervals on an icewater mixture. After centrifugation at $9400 \times g$ for 10 minutes, the supernatants were diluted with ChIP dilution buffer with PIC. One percentage of the total chromatin DNA was used as input. After incubation with anti-RCAN1 or anti-Flag antibody at $4{ }^{\circ} \mathrm{C}$ overnight, the supernatants were collected and then precleared with protein A/G-agarose beads. By vortexing at $1200 \mathrm{rpm} / \mathrm{min}$ for 30 minutes at $65^{\circ} \mathrm{C}$, chromatin was eluted from agarose beads. To reverse crosslinks, chromatin was incubated with $6 \mu \mathrm{l}$ of $5 \mathrm{M} \mathrm{NaCl}$ and $2 \mu \mathrm{l}$ of proteinase $\mathrm{K}$ at $65^{\circ} \mathrm{C}$ for $2 \mathrm{~h}$. DNA for PCR analysis was purified using a DNA spin column. 


\section{Luciferase assay}

NP cells were seeded in a 12-well plate at a density of $1 \times 10^{4}$ cells and transfected with or without equal amounts of pGL4 vector (Promega, WI, USA), SOX9Luc2 plasmid, or MMP13-Luc2 plasmid (Sunya, Hangzhou, China) with NFATc1 or HIF-1 $\alpha$ plasmid. Meanwhile, Renilla plasmid was cotransfected to normalize the transfection efficiency. After $24 \mathrm{~h}$, a luciferase assay was then carried out using a dual-luciferase reporter assay system (Beyotime).

\section{Isolation and ex vivo culture of rats lumbar IVDs}

Whole lumbar IVDs (NP, CEP, AF, and adjacent vertebral endplates) were gently harvested from rats (male, 150 200 g, 8 weeks old) as previously described ${ }^{31}$. They were cultured with DMEM, $50 \mu \mathrm{g} / \mathrm{mL}$ L-ascorbate and $10 \%$ fetal bovine serum and randomly divided into four groups and treated as follows $(n=6)$ : (A) IVDs+DMSO, (B) IVDs+FK506 $(1 \mu \mathrm{m}),(\mathrm{C})$ IVDs+DMOG $(1 \mu \mathrm{m})$, and (D) IVDs+DMOG $(1 \mu \mathrm{m})+$ FK506 $(1 \mu \mathrm{m})$. Treatments were administered for 2 weeks in six-well plates in a humid incubator $\left(37^{\circ} \mathrm{C}, 5 \% \mathrm{CO}_{2}\right)$. The expression levels of Sox9, type II collagen, MMP3, MMP13, ADAMTS4, and NFATc1 in the NP zone were measured using RTqPCR and immunofluorescence staining.

\section{Histological analysis}

Rat IVDs were fixed in $4 \%$ paraformaldehyde for $24 \mathrm{~h}$, decalcified in 12\% EDTA for 2 weeks, and then embedded in paraffin. For every IVD, slices $(4 \mu \mathrm{m}$-thick $)$ were obtained using a microtome (Leica, Germany). Slices were stained with safranin $\mathrm{O} /$ fast green or Alcian blue to examine cell morphology and ECM degeneration. Degenerative discs underwent detailed histological scoring (Supplemental Table 3) as previously described ${ }^{31}$. To examine RCAN1, MMP13 or type II collagen immunoreactivity, IHC or immunofluorescence staining was performed. Slices were photographed and assessed using a digital microscope.

\section{Statistical analysis}

The data are presented as the mean and standard deviation. Data analyses were conducted using SPSS 19.0 (SPSS, Chicago, IL, USA). Statistical differences were analyzed by one-way analysis of variance or Student's $t$ test, followed by Tukey's post hoc analysis when appropriate. Differences with a $P$ value $<0.05$ were considered statistically significant.

\section{Results}

\section{High RCAN1.4 in expression degenerated human NP} tissues

We divided patients into the Pfirrmann grade I/II, III, $\mathrm{IV}$, and $\mathrm{V}$ groups based on the Pfirrmann grading system $^{30}$. Representative H\&E, safranin $\mathrm{O} /$ fast green, and Alcian blue staining images of degenerative discs used to investigate the extent of degeneration are shown in Fig. 1a. We then detected gene expression of RCAN1.1, RCAN1.4, SOX9, type II collagen (Col2a1), aggrecan (ACAN), MMP13, and MMP3 in the four different groups using RT-qPCR (Fig. 1b). There was no obvious difference in the gene expression of RCAN1.1 or RCAN1.4 between the IDD group (grade IV and grade V) and the mild IDD group (grades I/II and grade III). Nevertheless, the RCAN1 protein level was visibly increased in the IDD group by immunohistochemistry (Fig. 1c). Furthermore, western blot assay showed that the protein level of RCAN1.4 was higher than that of RCAN1.1 and progressively elevated from the control group to the grade $\mathrm{V}$ group (Fig. 1d). In addition, we demonstrated upregulated RCAN1 and downregulated HIF- $1 \alpha$ levels in a tail-looping disc degeneration model in rats (Fig. 1e). These data indicated that RCAN1.4 is strongly associated with disc degeneration.

\section{RCAN1.4 was downregulated under hypoxic conditions}

Aggravation of IDD has been associated with downregulated expression of HIF- $1 \alpha$ but not HIF- $2 \alpha^{11,33}$. Thus, we detected RCAN1.4 expression under conditions of hypoxia or HIF- $1 \alpha$ overexpression to investigate the relationship between RCAN1.4 and relative hypoxia in NP cells. Gene expression of RCAN1.1 or RCAN1.4 was not significantly altered in human NP cells under hypoxic conditions or DMOG (a PHD inhibitor) treatment for $48 \mathrm{~h}$ (Fig. 2a and b). However, the RCAN1.4 protein level in human NP cells was visibly decreased in a dose- and time-dependent manner under hypoxia or DMOG treatment (Fig. 2c-f). In addition, the RCAN1.4 protein level in human NP cells was reduced with the overexpression of HIF-1 $\alpha$ (Fig. 2g). To further investigate the mechanism of RCAN1.4 downregulation, we stimulated human NP cells with MG132 (a proteasome inhibitor) or leupeptin (a lysosome inhibitor). As shown in Supplemental Fig. 1a and b, the change in RCAN1.4 expression was not reversed when the proteasome or lysosomal pathway was blocked. Furthermore, the downregulation of RCAN1 under hypoxia was not dependent on the ubiquitination degradation pathway (Supplemental Fig. 1c). We also predicted the microRNA-binding sites targeting RCAN1 and selected several microRNAs. Under hypoxia or DMOG treatment, rno-miR-124-3p was slightly increased (Fig. $2 \mathrm{~h}$ and i). In addition, overexpression of HIF- $1 \alpha$ upregulated the rno-miR-124-3p level (Fig. 2j), and rno-miR124-3p mimic suppressed RCAN1.4 expression (Fig. 2k). Together, these results show that RCAN1.4 was downregulated by increasing levels of rno-miR-124-3p under hypoxic conditions. 


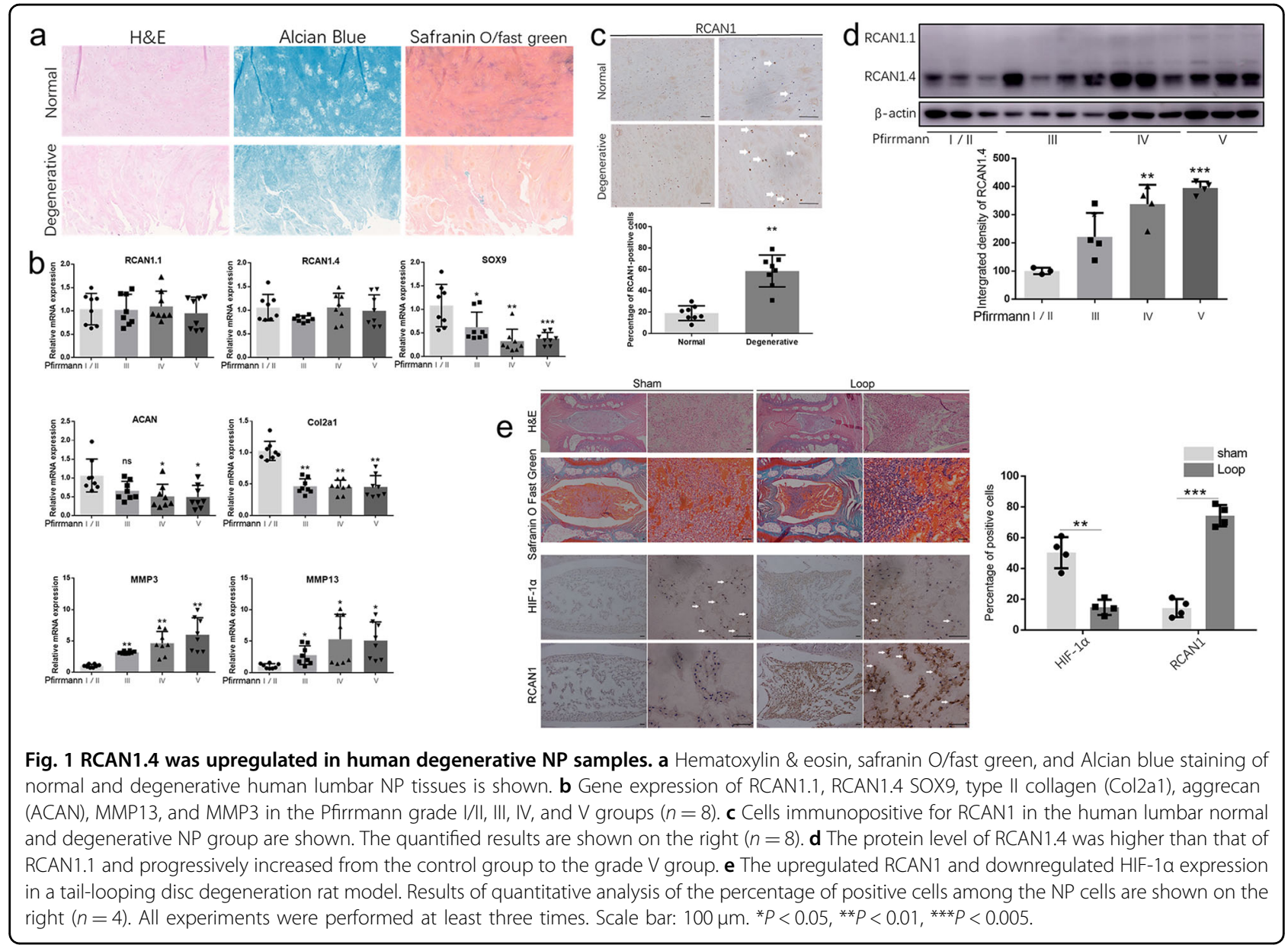

\section{Downregulated RCAN1 was accompanied with the} activation of calcineurin/NFAT under hypoxic conditions

As expected, the activity of calcineurin was upregulated under hypoxia (Fig. 2l). Because RCAN1.4 can specifically bind calcineurin at or near the calcineurin A catalytic domain and downregulate calcineurin activity ${ }^{14,15}$, we knocked down RCAN1.4 by using RCAN1.4 siRNA and detected the activity of calcineurin in rat NP cells. Notably, calcineurin A was activated in rat NP cells transfected with RCAN1.4 siRNA (Fig. 2m). Furthermore, IF and western blotting demonstrated that the nuclear translocation of NFATc1 was obviously increased in rat NP cells under hypoxia (Fig. 2n-p), indicating that the calcineurin/ NFAT signaling pathway is activated in hypoxic NP cells, mainly by the downregulation of RCAN1.4.

\section{RCAN1.4-calcineurin/NFAT signaling pathway altered ECM synthesis and remodeling}

To reveal the function of the RCAN1.4-calcineurin/NFAT signaling pathway in IDD, we used RCAN1.4 siRNA, RCAN1.4 overexpression plasmid, or FK506 (a calcineurin inhibitor) in rat NP cells under hypoxia. As shown in Fig. 3a and b, hypoxia-induced upregulation of SOX9 and
MMP13 was restrained by FK506. Furthermore, an immunofluorescence experiment showed that nuclear translocation of SOX9 and expression of type II collagen under hypoxia were sharply reduced by FK506 (Fig. 3c and d). Furthermore, RCAN1.4 overexpression reversed hypoxia-induced expression of SOX9 and MMP13 (Fig. 3 e) and inhibited the hypoxia-induced nuclear translocation of NFATc1 (Fig. 3f). In addition, RCAN1.4 knockdown by RCAN1.4 siRNA promoted the expression of SOX9 and MMP13, but their high expression levels were strongly suppressed by FK506 (Fig. 3g). Together, these data show that calcineurin/NFAT activation by hypoxiainduced downregulation of RCAN1.4 promotes ECM synthesis and remodeling.

\section{Nuclear translocation of NFATc1 promoted SOX9 and MMP13 promoter activity}

To illuminate the mechanism by which hypoxia promotes the transcription of SOX9 and MMP13, we first demonstrated that overexpression of NFATc1 upregulated SOX9 and MMP13 expression (Fig. 4a). We then cloned their promoter into a luciferase reporter plasmid. We first predicted putative NFATc1-binding sites within 


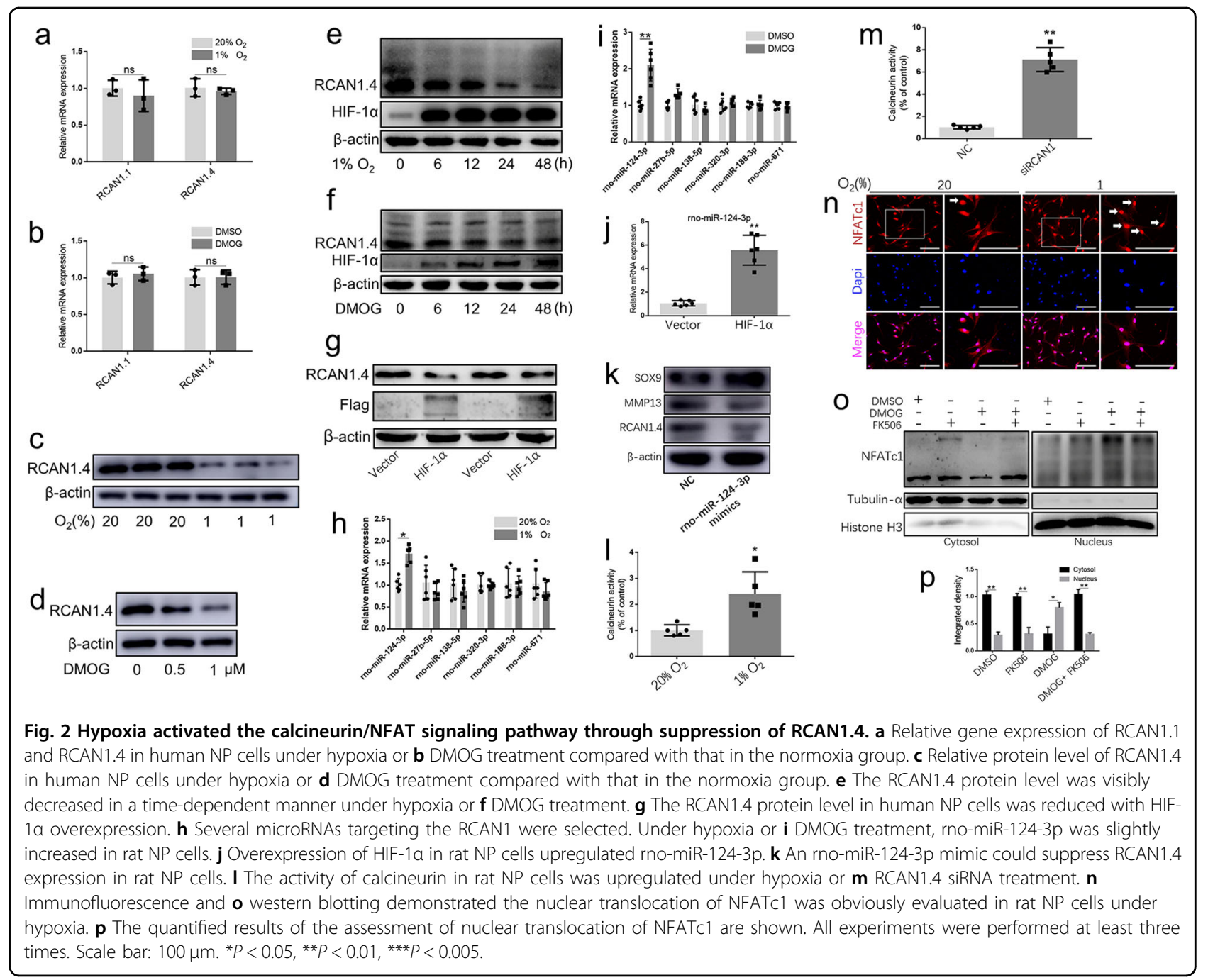

the SOX9 and MMP13 promoter regions with a length of 2000 bp with JASPAR: http://jaspar.genereg.net. We then generated five constructs derived from the $2000 \mathrm{bp}$ upstream of the rat SOX9 promoter (Fig. 4b). We detected robust NFATc1-drived luciferase activity with the P1, P3, and P4 fragments, but not the P2 and P5 fragments, in NP cells (Fig. 4c), indicating the presence of a positive regulatory element between $\mathrm{P} 4$ and $\mathrm{P} 5$. Further, to verify that NFATc1 regulates endogenous SOX9 transcription, we conducted a ChIP assay in NP cells. Surprisingly, NFATc1 increasingly bound the SOX9 promotor site (bp -470 to -323 ) in NP cells under hypoxia, whereas other binding sites $(\mathrm{bp}-1717$ to $-1610,-1484$ to -1370 and -1384 to -1273 ) were not elevated (Fig. 4d). Furthermore, we introduced mutations in the putative NFATc1-binding site of the P4 fragment (P4 mut1 and mut2) (Fig. 4e) and showed that P4 mutation decreased luciferase activity (Fig. 4f). Similarly, a ChIP assay demonstrated that NFATc1 could also bind sites in the MMP13 promotor (bp -1463 to -1361 and -321 to -216 ) in NP cells under hypoxia, while binding at the other binding site (bp -632 to -514) was not elevated (Fig. 4g). Surprisingly, we revealed that the interaction of HIF- $1 \alpha$ and NFATc1 positively regulated MMP13 transcription (Fig. 4h). In addition, accumulation of the of HIF- $1 \alpha$ and NFATc1 protein complex increased under hypoxia, which might facilitate their gene transcription (Fig. 4i).

\section{The role of the RCAN1.4-calcineurin/NFAT signaling pathway in an ex vivo model of rat discs}

To investigate the potential protective effects of RCAN1.4-calcineurin/NFAT in IVDDs, we generated an ex vivo model of rat lumbar discs. Safranin O/fast green and Alcian blue staining demonstrated the disc ECM in the control group was slightly degraded (Fig. 5a), as previously described ${ }^{34}$. In addition, DMOG treatment attenuated disc degeneration (Fig. 5a). FK506 (a calcineurin 


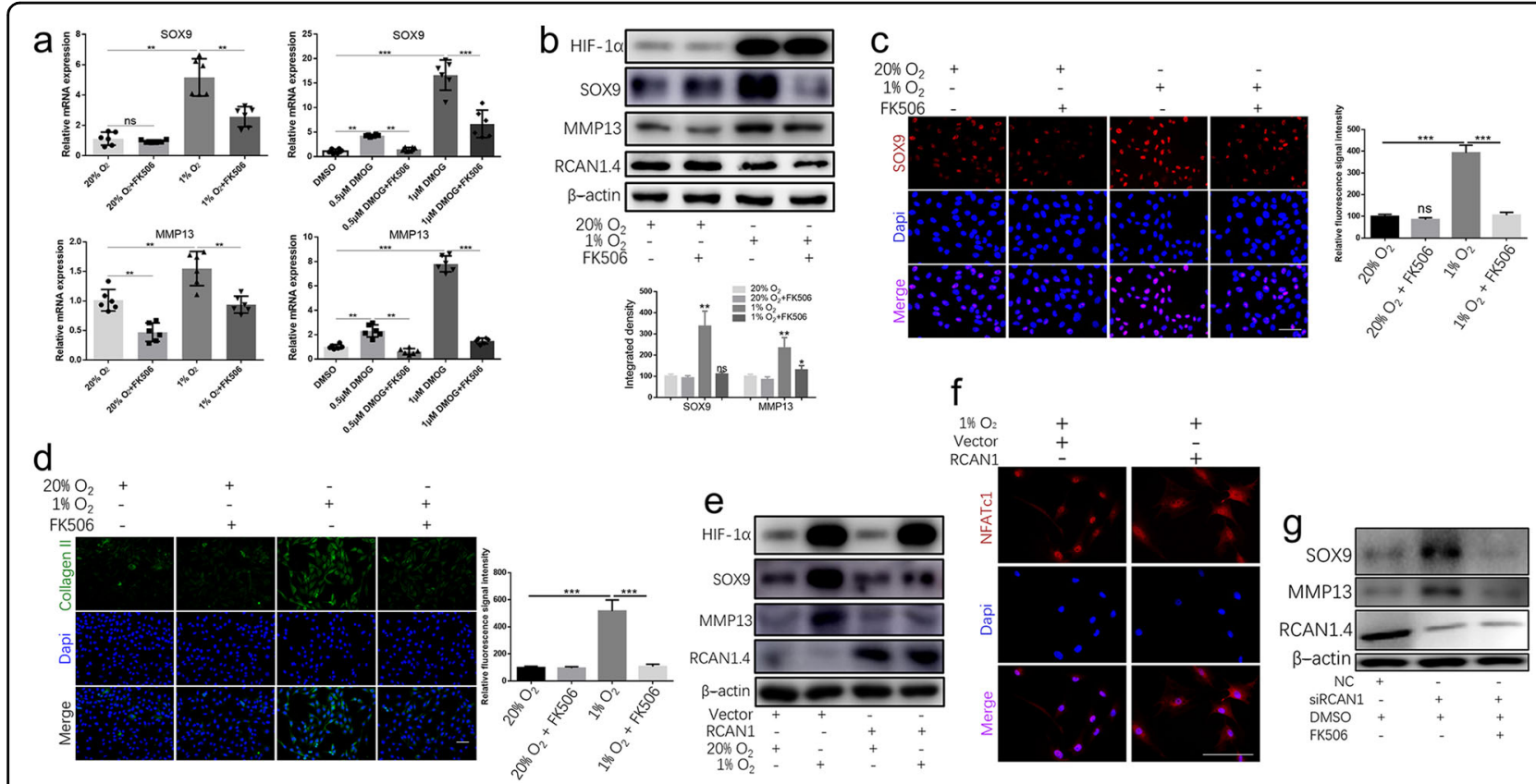

Fig. 3 RCAN1.4 the ECM synthesis and remodeling by regulating the calcineurin/NFAT signaling pathway. a Relative SOX9 and MMP13 gene expression in rat NP cells treated with or without FK506 (a calcineurin inhibitor) and stimulated with hypoxia or DMOG treatment. $\mathbf{b}$ Western blot analysis of rat NP cells treated with or without FK506 and stimulated with hypoxia or DMOG treatment. The quantified results are shown below. c Immunofluorescence analysis of SOX9 and $\mathbf{d}$ type II collagen in rat NP cells treated with or without FK506 and stimulated with hypoxia or DMOG treatment for $48 \mathrm{~h}$. Results of the quantification of SOX9 and type II collagen expression are shown on the right. e Western blot analysis of rat NP cells treated with vector or RCAN1.4 plasmid and stimulated with hypoxia or DMOG treatment. f Immunofluorescence demonstrated that RCAN1.4 overexpression suppressed the nuclear translocation of NFATC1 in rat NP cells under hypoxia. $\mathbf{g}$ Relative expression of SOX9 and MMP13 in rat NP cells treated with or without FK506 and stimulated with negative control siRNA or 100 nM RCAN1.4 siRNA. All experiments were performed at least three times. Scale bar: $100 \mu \mathrm{m} .{ }^{*} P<0.05,{ }^{* *} P<0.01,{ }^{* * *} P<0.005$.

inhibitor) counteracted the effect of DMOG on discs (Fig. $5 \mathrm{a}$ and $\mathrm{b}$ ). After DMOG treatment for 14 days, SOX9, type II collagen, MMP13, and NFATc1 expression was significantly increased (Fig. 5c). However, when discs were costimulated with FK506 and DMOG, SOX9, type II collagen, MMP13 expression was partially reversed to the levels in control cells (Fig. 5c). Immunohistochemistry showed decreased RCAN1 and increased HIF-1 $\alpha$ expression after DMOG or FK506 treatment (Fig. 5d). In addition, immunofluorescence revealed that DMOGinduced MMP13 and type II collagen expression could be suppressed by FK506 treatment (Fig. 5e and f). Finally, schematic representation of major molecular pathway was presented (Fig. 5g).

\section{Discussion}

Disc degeneration classification is carried out through subscoring of the following major subtissues: the NP, AF, and $\mathrm{CEP}^{35,36}$. If any of these structures is damaged or degenerated, the hypoxic environment of the disc will be destroyed. Understanding the underlying mechanisms that regulates ECM synthesis and the remodeling of disc cells under hypoxia is vital for future treatment. In this study, we first demonstrated that RCAN1.4 is positively associated with disc degeneration and that hypoxiainduced downregulation of RCAN1.4 activated the calcineurin/NFAT signaling pathway to facilitate SOX9 and MMP13 expression. Furthermore, NFATc1 could directly bind the promotor regions of SOX9 and MMP13. In addition, a whole disc ex vivo model showed that the RCAN1.4-calcineurin/NFAT signaling pathway is critical for the hypoxic IVD phenotype under hypoxia.

RCAN1.4 has a wide range of biological roles, including protecting against calcium-mediated oxidative stress $^{37}$, cardiac hypertrophy ${ }^{38}$, and vascular endothelial growth factor-mediated signaling during angiogenesis ${ }^{39}$. In addition, RCAN1 is more highly expressed than RCAN2 or RCAN3 in NP cells (data not shown). Therefore, we assumed that RCAN1 has a vital role in the IVD phenotype. Nevertheless, evidence regarding the role of RCAN1 in degenerative disc diseases is somewhat limited. We first showed that RCAN1.4 was upregulated with an increasing degree of disc degeneration. Although smaller vessels can infiltrate the cartilage endplates and outer AF layers, they cannot infiltrate the $\mathrm{NP}^{5}$. As a result, NP tissues under physiological conditions are a hypoxic microenvironment ${ }^{7}$. Cancer cells are also under a hypoxic microenvironment, and low expression levels of RCAN1.4 can 


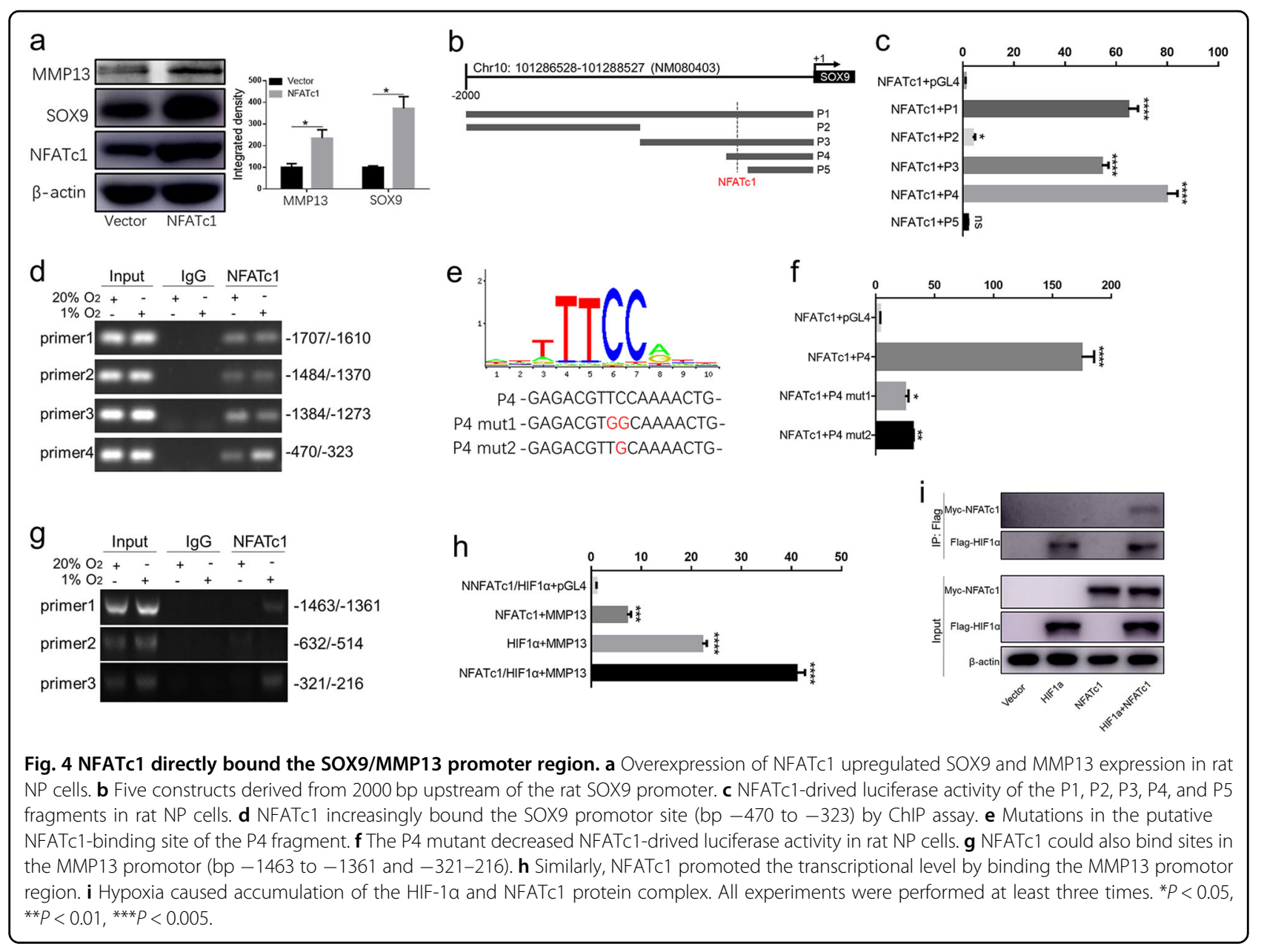

promote the proliferation, migration, and invasive activity of cancer cells by promoting nuclear translocation of NFAT $^{17}$. These studies indicated that RCAN1.4 participates in the hypoxic adaption of the IVD. Although calcineurin is activated by increased intracellular calcium ${ }^{40}$, we found that hypoxia inhibited RCAN1.4 protein expression, thus activating the calcineurin/NFAT signaling pathway and maintaining the hypoxic IVD phenotype. Degradation of RCAN1 was shown to be mediated by the ubiquitin-proteasome pathway ${ }^{41}$. However, our study demonstrated that downregulation of RCAN1.4 under hypoxia was not dependent on the ubiquitination degradation pathway. Nevertheless, we have not clarified the mechanism of RCAN1.4 downregulation, which might involve microRNA regulation.

Mature NP cells are small, chondrocyte-like cells sparsely distributed throughout the disc ${ }^{42}$. The transcription factor SOX9 is involved in chondrogenesis ${ }^{43}$. A recent study showed that a $\mathrm{CoCl}_{2}$-simulated hypoxic environment significantly increased the expression levels of calcineurin in mouse skeletal muscles ${ }^{44}$. Although hypoxia resulted in HIF- $1 \alpha$ accumulation, enhancing SOX9-mediated expression of the target gene type II collagen in femoral chondrocytes $^{26}$, it was still unclear whether the calcineurin/ NFAT signaling pathway is involved in the regulation of SOX9. Interestingly, FK506, a calcineurin inhibitor, suppressed the nuclear translocation of NFATc1, thus restraining SOX9 transcription and expression in NP cells. We detected the expression level of NFAT family members and found a high level of NFATc1 expression (data not shown). In addition, NFATc1 was positively associated with upregulated SOX9 in the pancreas and tracheal cartilage $^{23,24}$. We further revealed that NFATc1 enhanced the promoter activity of SOX9. By mutating the vital NFATc1binding site in the SOX9 promotor region, we then determined that its primary binding site is from bp -367 to -357 on the 2000 bp upstream of the rat SOX9 promoter. It is possible that hypoxia resulted in accumulation of the HIF- $1 \alpha$ and NFATc1 protein complex in hypoxic NP cells, assisting gene transcription.

PHD2 deficiency can activate the calcineurin/NFATc1dependent pathway ${ }^{21}$, indicating that HIF-1 $\alpha$ is possibly involved in activating the calcineurin/NFATc1 pathway. We found elevated calcineurin activity under hypoxia or 


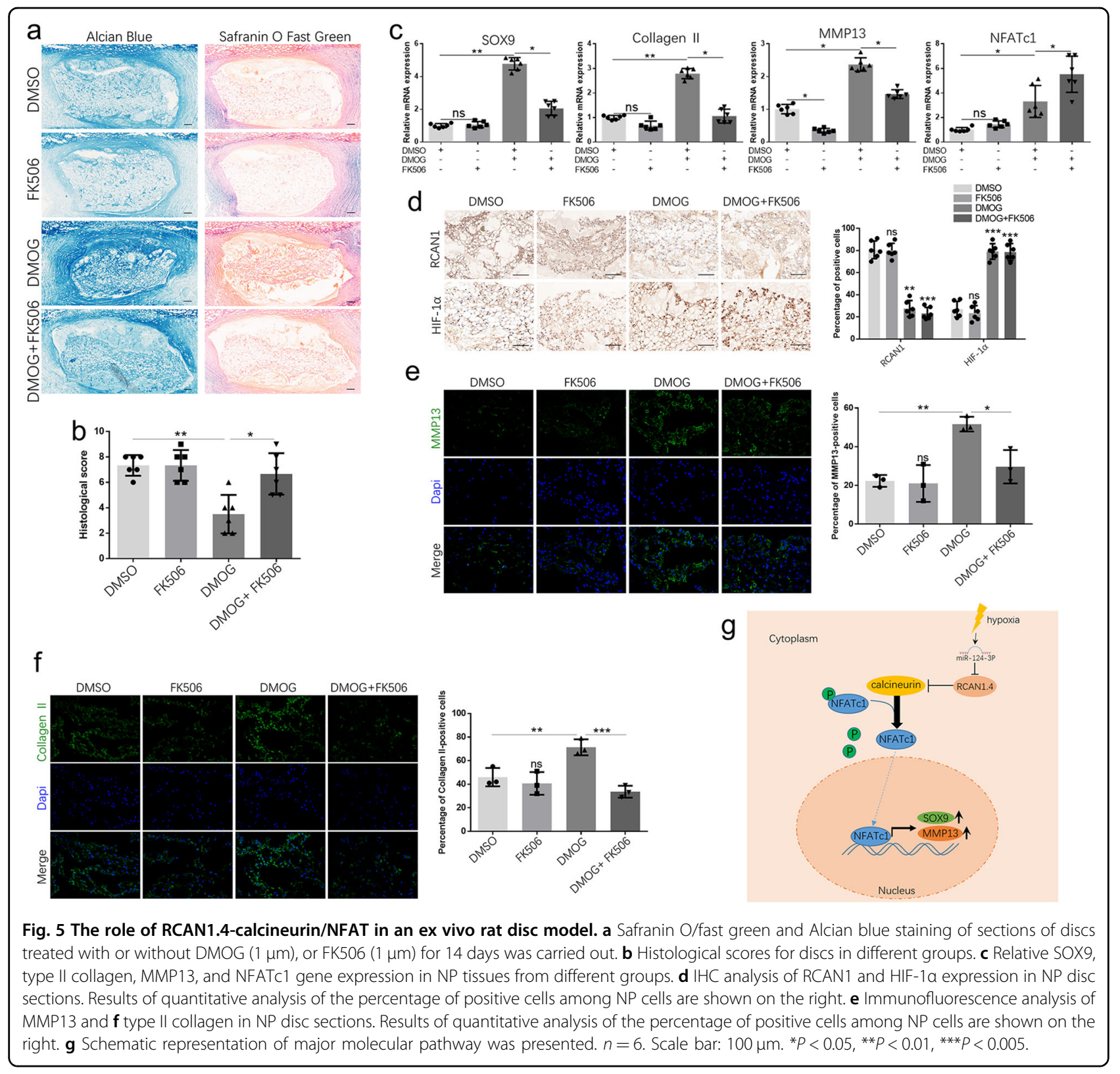

RCAN1.4 siRNA transfection, further promoting the nuclear translocation of NFATc1. Although independent silencing of calcineurin A (PPP3CA) expression stimulated HIF transcriptional activity under hypoxia ${ }^{45}$, we could not determine the regulated relationship between calcineurin and HIF- $1 \alpha$ based on our results. Hypoxiainduced mitogenic factor overexpression increased the cytosolic $\mathrm{Ca}^{2+}$ concentration and activated the calcineurin/NFAT pathways in cardiac hypertrophy ${ }^{29}$. Here, we detected the cytosolic $\mathrm{Ca}^{2+}$ concentration in hypoxic NP cells and found no noticeable change in the cytosolic $\mathrm{Ca}^{2+}$ concentration of NP cells (data not shown). Nevertheless, we showed the activation and inactivation of the calcineurin/NFATc1 signaling pathway by overexpression or blockade of RCAN1.4, respectively. Calcium influx could be associated with the RCAN1.4 protein level, but the relationship between calcium and RCAN1.4 in NP cells still requires further confirmation.

Hypoxia strongly promotes ECM synthesis, after which the ECM is remodeled and better organized to maintain homeostasis in the disc. We found slightly elevated levels ( 1.6-fold) of MMP13, a marker of ECM remodeling, under $1 \% \mathrm{O}_{2}$. A study reported that the interaction between $\beta$-catenin and HIF-1 $\alpha$ inhibited MMP13 expression and prohibited articular cartilage damage in mice $^{46}$. Hypoxia suppressed serum deprivation-induced ECM degradation of NP cells through the JNK and NF- $\mathrm{KB}$ pathways $^{47}$. Hypoxic isolation-expansion of human NP 
cells was important to achieve better regenerative cells for later cultivation or cell transplantation ${ }^{48}$. However, hypoxia was also shown to alter the gut microbiota, resulting in the senescence of bone marrow mesenchymal stem cells ${ }^{49}$. Therefore, hypoxia might be a "double-edged sword". We believe that hypoxia slightly remodels the ECM while hypoxia strongly promotes ECM synthesis in NP cells. Based on the results of the luciferase assay, NFATc1 strongly bound the MMP13 promotor region, thus upregulating MMP13 transcription. MMP13 expression could also be suppressed by FK506.

In conclusion, RCAN1.4 is involved in the hypoxiainduced IVD phenotype mainly through its regulation of the calcineurin/NFATc1 signaling pathway in NP cells. The calcineurin/NFATc1 signaling pathway is crucial for ECM synthesis and remodeling in discs. Our study shows that RCAN1.4 could be a potential therapeutic target for degenerative disc diseases. Further studies might focus on the effect of RCAN1.4 on AF or the cartilage endplates.

\section{Acknowledgements}

This work was supported by the Natural Science Foundation of China (no. 81871796) and the Zhejiang Provincial Natural Science Foundation of China (LQ19H060005).

\section{Author details}

'Department of Orthopedic Surgery, Sir Run Run Shaw Hospital, Zhejiang University School of Medicine, Hangzhou, China. ${ }^{2}$ Key Laboratory of Musculoskeletal System Degeneration and Regeneration Translational Research of Zhejiang Province, Hangzhou, China. ${ }^{3}$ Department of Orthopedic Surgery, Haining People's Hospital, Haining, China

\section{Author contributions}

Study design: $\mathrm{BH}, \mathrm{YQH}, \mathrm{JC}$, and FDZ. Study execution: $\mathrm{BH}, \mathrm{YQH}$, and SWL. Data collection and analysis: BH, YQH, JHL, and XAW. Data interpretation: JC, ZS, and FDZ. Drafting of the manuscript: $\mathrm{BH}, \mathrm{YH}$, and JC. Revision of the manuscript: JC and FDZ. All authors take responsibility for the integrity of the data analysis. All authors were involved in the study conception and design.

\section{Conflict of interest}

The authors declare that they have no conflict of interest.

\section{Publisher's note}

Springer Nature remains neutral with regard to jurisdictional claims in published maps and institutional affiliations.

Supplementary information accompanies this paper at https:/doi.org/ 10.1038/s12276-020-0441-x.

Received: 7 December 2019 Accepted: 15 April 2020.

Published online: 29 May 2020

\section{References}

1. Deyo, R. A. \& Weinstein, J. Low back pain. N. Engl. J. Med. 344, 363-370 (2001).

2. Chou, R. \& Shekelle, P. Will this patient develop persistent disabling low back pain? JAMA 303, 1295-1302 (2010).

3. Broberg, K. On the mechanical behaviour of intervertebral discs. Spine $\mathbf{8}$, 151-165 (1983).

4. Setton, L. A. \& Chen, J. Mechanobiology of the intervertebral disc and relevance to disc degeneration. J. Bone Jt. Surg. Am. Vol. 88, 52-57 (2006).
5. Kepler, C. K., Ponnappan, R. K, Tannoury, C. A., Risbud, M. V. \& Anderson, D. G. The molecular basis of intervertebral disc degeneration. Spine J. 13, 318-330 (2013).

6. Oegema, T. R. Biochemistry of the intervertebral disc. Clin. Sports Med. 12 419-439 (1993).

7. Urban, J. P. G., Smith, S. \& Fairbank, J. C. T. Nutrition of the intervertebral disc. Spine 29, 2700-2709 (2004).

8. Semenza, G. L., Roth, P. H., Fang, H. M. \& Wang, G. L. Transcriptional regulation of genes encoding glycolytic enzymes by hypoxia-inducible factor 1. J. Biol. Chem. 269, 23757-23763 (1994).

9. Silagi, E. S. et al. Bicarbonate recycling by HIF-1-dependent carbonic anhydrase isoforms 9 and 12 is critical in maintaining intracellular $\mathrm{pH}$ and viability of nucleus pulposus cells. J. Bone Miner. Res. 33, 338-355 (2018).

10. Zhang, B., Zhao, Q., Li, Y. \& Zhang, J. Moxibustion alleviates intervertebral disc degeneration activation of the HIF-1aNEGF pathway in a rat model. Am. J. Transl. Res. 11, 6221-6231 (2019).

11. Chen, S. et al. PHD/HIF-1 upregulates CA12 to protect against degenerative disc disease: a human sample, in vitro and ex vivo study. Lab. Investig. 96, 561-569 (2016).

12. Liu, Z. et al. Hypoxia-inducible factor-lalpha mediates aggrecan and collagen P expression via NOTCH1 signaling in nucleus pulposus cells during intervertebral disc degeneration. Biochem. Biophys. Res. Commun. 488, 554-561 (2017).

13. Davies, K. J. et al. Renaming the DSCR1/Adapt78 gene family as RCAN: regulators of calcineurin. FASEB J. 21, 3023-3028 (2007).

14. Rothermel, B. et al. A protein encoded within the Down syndrome critical region is enriched in striated muscles and inhibits calcineurin signaling. J. Biol. Chem. 275, 8719-8725 (2000).

15. Fuentes, J. J. et al. DSCR1, overexpressed in Down syndrome, is an inhibitor of calcineurin-mediated signaling pathways. Hum. Mol. Genet. 9, 1681-1690 (2000).

16. Wang, C. et al. RCAN1-4 is a thyroid cancer growth and metastasis suppressor JCl Insight 2, e90651 (2017).

17. Jin, H. et al. Regulator of calcineurin 1 gene isoform 4, downregulated in hepatocellular carcinoma, prevents proliferation, migration, and invasive activity of cancer cells and growth of orthotopic tumors by inhibiting nuclear translocation of NFAT1. Gastroenterology 153, 799-811 (2017).

18. Alghanem, A. F. et al. RCAN1.4 regulates VEGFR-2 internalisation, cell polarity and migration in human microvascular endothelial cells. Angiogenesis $\mathbf{2 0}$ 341-358 (2017)

19. Villahoz, S. et al. Conditional deletion of Rcan1 predisposes to hypertensionmediated intramural hematoma and subsequent aneurysm and aortic rupture. Nat. Commun. 9, 4795 (2018).

20. Yun, Y. et al. Regulator of calcineurin 1 is a novel RNA-binding protein to regulate neuronal apoptosis. Mol. Psychiatry https://doi.org/10.1038/s41380019-0487-0 (2019).

21. Shin, J. et al. Prolyl hydroxylase domain 2 deficiency promotes skeletal muscle fiber-type transition via a calcineurin/NFATc1-dependent pathway. Skelet. Muscle 6, 5 (2016)

22. Fujita, N. et al. HIF-1-PHD2 axis controls expression of syndecan 4 in nucleus pulposus cells. FASEB J. 28, 2455-2465 (2017).

23. Tzeng, B. H., Lee, K. R., Smith, R. J., Campbell, K. P. \& Chen, C. Cav3.2 T-type calcium channel is required for the NFAT-dependent Sox9 expression in tracheal cartilage. Proc. Natl Acad. Sci. USA 111, E1990-E1998 (2014).

24. Chen, N. M. et al. NFATc1 links EGFR signaling to induction of Sox9 transcription and acinar-ductal transdifferentiation in the pancreas. Gastroenterology 148, 1024-1034 e1029 (2015).

25. Bradley, E. W. \& Drissi, M. H. WNT5A regulates chondrocyte differentiation through differential use of the CaN/NFAT and IKK/NF-kappaB pathways. Mol. Endocrinol. 24, 1581-1593 (2010).

26. Zhang, C. et al. Hypoxia-inducible factor-1 is a positive regulator of Sox9 activity in femoral head osteonecrosis. Bone 48, 507-513 (2011).

27. Stegen, $\mathrm{S}$. et al. HIF-1alpha metabolically controls collagen synthesis and modification in chondrocytes. Nature 565, 511-515 (2019).

28. Walczak-Drzewiecka, A., Ratajewski, M., Wagner, W. \& Dastych, J. HIF-1 is upregulated in activated mast cells by a process that involves calcineurin and NFAT. J. Immunol. 181, 1665-1672 (2008).

29. Kumar, S. et al. Hypoxia-induced mitogenic factor promotes cardiac hypertrophy via calcium-dependent and hypoxia-inducible factor-1a mechanisms. Hypertension 72, 331-342 (2018). 
30. Pfirrmann, C. W. A., Metzdorf, A., Zanetti, M., Hodler, J. \& Boos, N. Magnetic resonance classification of lumbar intervertebral disc degeneration. Spine $\mathbf{2 6}$, 1873-1878 (2001).

31. Xie, Z. et al. TGF-beta synergizes with ML264 to block IL-1beta-induced matrix degradation mediated by Kruppel-like factor 5 in the nucleus pulposus. Biochim. Biophys. Acta 1864, 579-589 (2018).

32. Huang, B. et al. Administration of SB239063 Ameliorates Ovariectomy-Induced Bone Loss via Suppressing Osteoclastogenesis in Mice. Front. Pharmacol. 10, 900 (2019).

33. Ha, K. Y. et al. The expression of hypoxia inducible factor-1alpha and apoptosis in herniated discs. Spine 31, 1309-1313 (2006)

34. Haschtmann, D., Stoyanov, J. V., Ettinger, L., Nolte, L. P. \& Ferguson, S. J. Establishment of a novel intervertebral disc/endplate culture model: analysis of an ex vivo in vitro whole-organ rabbit culture system. Spine 31, 2918-2925 (2006).

35. Zehra, U., Noel-Barker, N., Marshall, J., Adams, M. A. \& Dolan, P. Associations between intervertebral disc degeneration grading schemes and measures of disc function. J. Orthop. Res. https://doi.org/10.1002/ jor.24326 (2019).

36. Boos, N. et al. Classification of age-related changes in lumbar intervertebral discs: 2002 Volvo Award in basic science. Spine 27, 2631-2644 (2002).

37. Lin, H. Y. et al. Oxidative and calcium stress regulate DSCR1 (Adapt78/MCIP1) protein. Free Radic. Biol. Med. 35, 528-539 (2003).

38. van Rooij, E. et al. MCIP1 overexpression suppresses left ventricular remodeling and sustains cardiac function after myocardial infarction. Circ. Res. 94, e18-e26 (2004).

39. Hesser, B. A. et al. Down syndrome critical region protein 1 (DSCR1), a novel VEGF target gene that regulates expression of inflammatory markers on activated endothelial cells. Blood 104, 149-158 (2004).
40. Crabtree, G. R. Generic signals and specific outcomes: signaling through Ca2+ calcineurin, and NF-AT. Cell 96, 611-614 (1999).

41. Liu, H., Wang, P., Song, W. \& Sun, X. Degradation of regulator of calcineurin 1 (RCAN1) is mediated by both chaperone-mediated autophagy and ubiquitin proteasome pathways. FASEB J. 23, 3383-3392 (2009).

42. Saggese, T., Redey, P. \& McGlashan, S. R. Same-species phenotypic comparison of notochordal and mature nucleus pulposus cells. Eur. Spine J. 24, 1976-1985 (2015).

43. Bi, W., Deng, J., Zhang, Z., Behringer, R. R. \& Crombrugghe, B. d. Sox9 is required for cartilage formation. Nat. Genet. 22, 85-89 (1999).

44. Li, L. et al. Effect of hypoxia on the muscle fiber switching signal pathways CnA/NFATC1 and myostatin in mouse myocytes. Acta Histochem. 121, 539-545 (2019).

45. Karagiota, A., Mylonis, I., Simos, G. \& Chachami, G. Protein phosphatase PPP3CA (calcineurin A) down-regulates hypoxia-inducible factor transcriptional activity. Arch. Biochem. Biophys. 664, 174-182 (2019).

46. Bouaziz, W. et al. Interaction of HIF1a and $\beta$-catenin inhibits matrix metalloproteinase 13 expression and prevents cartilage damage in mice. Proc. Natl Acad. Sci. 113, 5453-5458 (2016).

47. Wang, J. et al. Hypoxia suppresses serum deprivation-induced degradation of the nucleus pulposus cell extracellular matrix through the JNK and NF-kappaB pathways. J. Orthop. Res. 35, 2059-2066 (2017).

48. Yang, S. H. et al. The influence of oxygen concentration on the extracellular matrix production of human nucleus pulposus cells during isolation-expansion process. J. Biomed. Mater. Res. Part A 105, 1575-1582 (2017).

49. Xing, J. et al. Hypoxia induces senescence of bone marrow mesenchymal stem cells via altered gut microbiota. Nat. Commun. 9 , 2020 (2018). 